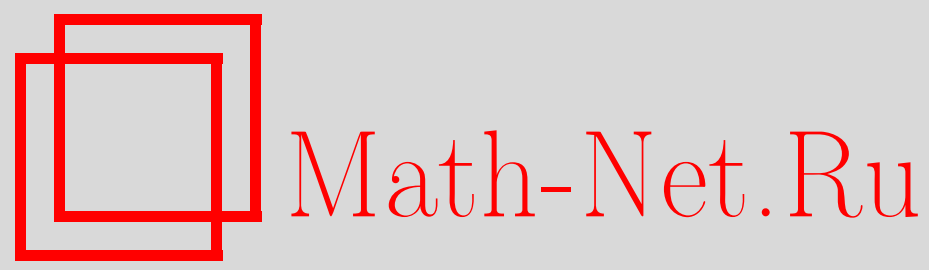

А. Ю. Колесов, Четырехмерный аналог одной классической задачи Ляпунова об устойчивости, Матем. заметки, 1996, том 60, выпуск 4, 612-615

DOI: https://doi.org/10.4213/mzm1871

Использование Общероссийского математического портала MathNet.Ru подразумевает, что вы прочитали и согласны с пользовательским соглашением

http://www . mathnet.ru/rus/agreement

Параметры загрузки:

IP : 54.210.77.194

26 апреля 2023 г., 14:21:11

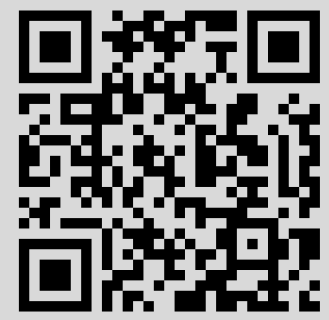




\section{ЧЕТЫРЕХМЕРНЫЙ АНАЛОГ ОДНОЙ КЛАССИЧЕСКОЙ ЗАДАЧИ ЛЯПУНОВА ОБ УСТОЙЧИВОСТИ}

\section{А. Ю. Колесов}

1. Будем интересоваться устойчивостью нулевого состояния равновесия четырехмерной системы

$$
\dot{u}=F(u), \quad F \in C^{\infty}\left(\mathbb{R}^{4} ; \mathbb{R}^{4}\right), \quad F(0)=0,
$$

в которой матрица $A=F^{\prime}(0)$ имеет чисто мнимые собственные значения $\pm i$ с клетками Жордана, т.е. $A e_{0}=i e_{0}, A e_{1}=i e_{1}+e_{0}$, где $e_{0}, e_{1} \in \mathbb{C}^{4}, e_{0} \neq 0$. В связи с этим сначала с помощью замены вида

$$
u=z e_{0}+\bar{z} \bar{e}_{0}+w e_{1}+\bar{w} \bar{e}_{1}+\Phi(z, w)
$$

где $z, w$ - комплексные переменные, приведем данную систему к нормальной форме Пуанкаре-Дюлака

$$
\begin{gathered}
\dot{z}=i z+w \\
\dot{w}=i w+a_{1}|z|^{2} z+a_{2}|z|^{2} w+a_{3} z^{2} \bar{w} \\
+a_{4}|w|^{2} z+a_{5} w^{2} \bar{z}+a_{6}|w|^{2} w+\Psi(z, w) .
\end{gathered}
$$

Здесь вектор-функция $\Phi$ и комплекснозначная функция $\Psi$ имеют в нуле тейлоровские разложения, начинающиеся с квадратичных членов и членов пятой степени соответственно, $a_{j}, j=1, \ldots, 6,-$ комплексные коэфффициенты нормальной формы, алгоритм нахождения которых приведен, например, в [1].

В монографии [2] доказана неустойчивость нулевого решения системы (1), если в нормальной форме $a_{1} \neq-b, b>0$. Поэтому естественно возникает задача (аналогичная классической задаче Ллпунова - двойной нулевой корень с клеткой) о получении критерия его устойчивости при условиях

$$
\operatorname{Im} a_{1}=0, \quad \operatorname{Re} a_{1}=-b, \quad b>0 .
$$

В формулируемом ниже утверждении $x=\operatorname{Re} a_{2}, y=\operatorname{Re} a_{3}$.

Теорема. Пусть выполнено условие (4). Тогда нулевое состояние равновесия системы (1) асимптотически устойчиво при $x<0,|y|<|x|$. Если же хотя би одно из этих неравенств меняет знак, то состояние равновесия неустойчиво.

Работа вьполненапри финансовой поддержке Российского фонда фундаментальных исследований. 
2. При доказательстве теоремы рассмотрим систему (3) на множестве $|z| \leqslant \delta$, $|w| \leqslant \delta^{2}$, где $\delta>0$ достаточно мало, и вьполним в ней последовательно замены

$$
z \exp (-i t) \rightarrow z, \quad w \exp (-i t) \rightarrow w, \quad \frac{z}{\delta} \rightarrow z, \quad \frac{z}{\delta^{2}} \rightarrow w, \quad \delta t \rightarrow t
$$

(первые две из них убирают вращение в линейной части, а остальные - удобные нормировки [2]). В итоге получаем систему

$$
\begin{gathered}
\dot{z}=w \\
\dot{w}=-b|z|^{2} z+\delta\left(a_{2}|z|^{2} w+a_{3} z^{2} \bar{w}\right) \\
+\delta^{2}\left(a_{4}|w|^{2} z+a_{5} w^{2} \bar{z}\right)+\delta^{3} a_{6}|w|^{2} w+\delta^{2} \Lambda(z, w, t, \delta)
\end{gathered}
$$

в которой, что вполне естественно, будем считать $\delta \ll 1$. Преимущество системы $(6)$ перед исходной заключается в том, что, во-первых, при $\delta=0$ она допускает первые интегралы

$$
\frac{b}{2}|z|^{4}+|w|^{2}=c_{1}, \quad \operatorname{Re}(i z \bar{w})=c_{2}, \quad c_{1}>0
$$

во-вторых, в силу нормировок из (5) онарассматривается в независящей от $\delta$ окрестности $B=\{(z, w):|z| \leqslant 1,|w| \leqslant 1\}$.

При $x \pm y<0$ (эти неравенства эквивалентны приведенньп в теореме) обыграем существование у системы (6) при $\delta=0$ первого семейства интегралов (7): функцию Ляпунова для нее будем искать в виде

$$
V=\frac{b}{2}|z|^{4}+|w|^{2}+2 \delta \operatorname{Re}\left[\alpha|z|^{2} z \bar{w}-\beta|w|^{2} w \bar{z}\right], \quad \alpha=\alpha_{1}+i \alpha_{2}
$$

где $\alpha_{2}=-\operatorname{Im} a_{3}$, параметр $\alpha_{1}>0$ выбран так, что

$$
x+y+3 \alpha_{1}<0, \quad x-y+\alpha_{1}<0,
$$

а выбором $\beta>0$ распорядимся позже. Непосредственная проверка показьвает, что производная функции (8) в силу системы (6) имеет вид

$$
\dot{V}=\delta f_{1}+2 \operatorname{Re}\left[\delta^{2} f_{2}+\delta^{3} f_{3}+\delta^{4} f_{4}+\delta^{2} \frac{\partial V}{\partial w} \Lambda\right],
$$

где

$$
\begin{aligned}
f_{1}= & -2 b \alpha_{1}|z|^{6}+\left(2 \operatorname{Re} a_{2}+4 \alpha_{1}\right)|z|^{2}|w|^{2}+2 \operatorname{Re}\left[\left(\alpha+a_{3}\right) z^{2} \bar{w}^{2}\right] \\
& -2 \beta|w|^{4}+4 b \beta|w|^{2}|z|^{4}+2 b \beta \operatorname{Re}\left(\bar{w}^{2}|z|^{2} z^{2}\right) \\
f_{2}= & \bar{w}\left(a_{4}|w|^{2} z+a_{5} w^{2} \bar{z}\right)+\left(\bar{\alpha} \bar{z}^{2} z-2 \beta|w|^{2} \bar{z}-\beta \bar{w}^{2} z\right)\left(a_{2}|z|^{2} w+a_{3} z^{2} \bar{w}\right), \\
f_{3}= & a_{6}|w|^{4}+\left(\bar{\alpha} \bar{z}^{2} z-2 \beta|w|^{2} \bar{z}-\beta \bar{w}^{2} z\right)\left(a_{4}|w|^{2} z+a_{5} w^{2} \bar{z}\right) \\
f_{4}= & a_{6}|w|^{2} w\left(\bar{\alpha} \bar{z}^{2} z-2 \beta|w|^{2} \bar{z}-\beta \bar{w}^{2} z\right) .
\end{aligned}
$$

Для анализа знака правой части формулы (10) на множестве $B$ сначала отбросим в формуле (11) последние два слагаемых, а в оставшейся части выполним замены

$$
z=\xi_{1} \exp \left(i \varphi_{1}\right), \quad w=\xi_{2} \exp \left(i \varphi_{2}\right), \quad \xi_{j} \geqslant 0, \quad j=1,2 .
$$


В результате получаем выражение

$$
f_{1}^{0}=-2 b \alpha_{1} \xi_{1}^{6}+\left[2 x+4 \alpha_{1}+2\left(y+\alpha_{1}\right) \cos 2 \psi\right] \xi_{1}^{2} \xi_{2}^{2}-2 \beta \xi_{2}^{4}
$$

где $\psi=\varphi_{2}-\varphi_{1}$. Заметим, что $f_{1}^{0}<0$ при $\xi_{1}+\xi_{2}>0,0 \leqslant \psi \leqslant \pi$, так как неравенства (9) обеспечивают отрицательность второго слагаемого в правой части (16). Опираясь, далее, на оценку

$$
\left.|4 b \beta| w\right|^{2}|z|^{4}+\left.2 b \beta \operatorname{Re}\left(\bar{w}^{2}|z|^{2} z^{2}\right)|\leqslant 6 b \beta| z\right|^{4}|w|^{2},
$$

убеждаемся, что при подходящем уменьшении $\beta$ знаки $f_{1}^{0}$ и $f_{1}$ на множестве $B$ совпадут.

Следующий этап связан с оценками

$$
\begin{array}{ll}
\left|f_{2}\right| \leqslant N\left(|w|^{3}|z|+|w| \cdot|z|^{5}\right), & \left|f_{3}\right| \leqslant N\left(|w|^{4}+|w|^{2}|z|^{4}\right), \\
\left|f_{4}\right| \leqslant N\left(|w|^{3}|z|^{3}+|w|^{5}|z|\right), & |\Lambda| \leqslant N(|z|+\delta|w|)^{5}
\end{array}
$$

вытекающими очевидным образом из формул (12)-(14) и отмеченных выше свойств остатка $\Psi$ в (3). Здесь постоянная $N>0$ не зависит от $t, \delta$ и $(z, w) \in B$. Учитывая эти неравенства вместе с оценками

$$
\begin{gathered}
|w|^{3}|z| \leqslant \frac{1}{2}\left(|w|^{4}+|w|^{2}|z|^{2}\right), \quad|w| \cdot|z|^{5} \leqslant \frac{1}{2}\left(|z|^{8}+|w|^{2}|z|^{2}\right) \\
|w|^{5}|z| \leqslant \frac{1}{2}\left(|w|^{8}+|w|^{2}|z|^{2}\right)
\end{gathered}
$$

в формуле (10), убеждаемся в совпадении знаков $\dot{V}$ и $f_{1}$ на множестве $B$. В части устойчивости теорема доказана.

При $x+y>0, x-y<0$ обыграем существование при $\delta=0$ второго семейства интегралов (7): рассмотрим функцию

$$
H=\operatorname{Re}(i z \bar{w})-\delta\left(\operatorname{Im} a_{2}+\operatorname{Im} a_{3}\right) \frac{|z|^{4}}{4}
$$

и криволинейный конус

$$
K=\left\{(z, w):|H| \leqslant \nu_{0} V, 0<V \leqslant \nu_{1}\right\} .
$$

Здесь параметры $\nu_{0}, \nu_{1}>0$ достаточно малы, а функция $V$ по-прежнему определяется формулой (8). Однако, теперь параметр $\alpha_{1}<0$ в ней выбран так, что $x+y+3 \alpha_{1}>0, \mathrm{a} \beta<0$ произвольно фиксировано. Отметим, что для производной $H$ в силу системы (6) справедливы неравенства

$$
\left.\dot{H}\right|_{H=\nu_{0} V, 0<V \leqslant \nu_{1}}<0,\left.\quad \dot{H}\right|_{H=-\nu_{0} V, 0<V \leqslant \nu_{1}}>0,
$$

а для функции $V$ в данном случае имеем

$$
\left.\dot{V}\right|_{(z, w) \in K}>0
$$

Действительно, оценки (21) элементарно следуют из формулы

$$
\dot{H}=\delta|z|^{2}(x-y) H+\delta^{2} R(z, w, t, \delta),
$$


в которой остаток

$$
R=|z|^{6}(x-y) \frac{\left(\operatorname{Im} a_{2}+\operatorname{Im} a_{3}\right)}{4}+\operatorname{Re}\left[i z\left(\bar{a}_{4}|w|^{2} \bar{z}+\bar{a}_{5} \bar{w}^{2} z+\delta \bar{a}_{6}|w|^{2} \bar{w}+\bar{\Lambda}\right)\right]
$$

удовлетворяет, очевидно, оценке

$$
|R| \leqslant N\left(|z|(|z|+|w|)^{5}+|w|^{2}|z|^{2}+|w|^{3}|z|\right) .
$$

В справедливости же же неравенства (22) убеждаемся на основе анализа формул (10)-(16), вполне аналогичного изложенному выше при доказательстве устойчивости (в данном случае $f_{1}^{0}>0$ в конусе $\left.(20)\right)$.

Из неравенств $(21),(22)$ следует, что траектории системы (6) входят внутрь конуса (20) через боковые поверхности $H= \pm \nu_{0} V$. В самом же конусе имеется функция $V$, положительно определенная вместе с производной в силу системы. Поэтому неустойчивость нулевого состояния в рассматриваемом случае - следствие простейшей геометрической теоремы из [2].

Случай $x-y>0, x+y<0$ практически полностью аналогичен разобранному выше. В качестве конуса (20) здесь возьмем

$$
K=\left\{(z, w): H \geqslant \nu_{0} V, 0<V \leqslant \nu_{1}\right\},
$$

где по-прежнему $\nu_{0}, \nu_{1}>0$ достаточно малы, а функции $V$ и $H$ определены формулами (8), (19). Однако, теперь в (8) параметр $\alpha_{1}>0$ выбран так, что $x+y+3 \alpha_{1}<0$, а параметр $\beta>0$ произвольно фиксирован. Рассуждая как и выше, в данном случае вместо (21), (22) получаем неравенства

$$
\left.\dot{V}\right|_{H=\nu_{0} V, 0<V \leqslant \nu_{1}}<0,\left.\quad \dot{H}\right|_{(z, w) \in K}>0
$$

из которых очевидньм образом вытекает неустойчивость нулевого состояния равновесия системы (6).

Для завершения доказательства теоремы остается добавить, что случай $x \pm y>0$ сводится к случаю $x \pm y<0$ заменами $t$ на $-t, w$ на $-w$, в результате которых $\operatorname{Re} a_{2}$ и $\operatorname{Re} a_{3}$ меняют знак.

Ярославский государственный университет

Поступило 15.04.96

\section{СПИСОК ЦИТИРОВАННОЙ ЛИТЕРАТУРЫ}

1. Колесов Ю. С. / / Исследования по устойчивости и теории колебаний. Ярославль, 1979. С. 3-40. 2. Хазин Л. Г., Шноль Э. Э. Устойчивость критических положений равновесия. Пущино: ОНТИ НЦБИ АН СССР, 1985. 